\title{
One-Step Leapfrog LOD-BOR-FDTD Algorithm with CPML Implementation
}

\author{
Yi-Gang Wang, ${ }^{1}$ Yun Yi, ${ }^{1}$ Bin Chen, ${ }^{1}$ Hai-Lin Chen, ${ }^{1}$ Kang Luo, ${ }^{1}$ and Run Xiong ${ }^{2}$ \\ ${ }^{1}$ PLA University of Science and Technology, Nanjing, Jiangsu 210007, China \\ ${ }^{2}$ Engineering Academy of PLA, Xuzhou, Jiangsu 221004, China \\ Correspondence should be addressed to Yun Yi; yiyun_rachel@sohu.com
}

Received 5 January 2016; Revised 12 March 2016; Accepted 14 April 2016

Academic Editor: Marta Cavagnaro

Copyright (c) 2016 Yi-Gang Wang et al. This is an open access article distributed under the Creative Commons Attribution License, which permits unrestricted use, distribution, and reproduction in any medium, provided the original work is properly cited.

\begin{abstract}
An unconditionally stable one-step leapfrog locally one-dimensional finite-difference time-domain (LOD-FDTD) algorithm towards body of revolution (BOR) is presented. The equations of the proposed algorithm are obtained by the algebraic manipulation of those used in the conventional LOD-BOR-FDTD algorithm. The equations for $z$-direction electric and magnetic fields in the proposed algorithm should be treated specially. The new algorithm obtains a higher computational efficiency while preserving the properties of the conventional LOD-BOR-FDTD algorithm. Moreover, the convolutional perfectly matched layer (CPML) is introduced into the one-step leapfrog LOD-BOR-FDTD algorithm. The equation of the one-step leapfrog CPML is concise. Numerical results show that its reflection error is small. It can be concluded that the similar CPML scheme can also be easily applied to the one-step leapfrog LOD-FDTD algorithm in the Cartesian coordinate system.
\end{abstract}

\section{Introduction}

The body of revolution finite-difference time-domain (BORFDTD) algorithm is very efficient in analyzing electromagnetic problems towards rotationally symmetric structures $[1$, 2]. It has been widely used in modeling electromagnetic pulse effects, electromagnetic wave scattering, subsurface interface radar, optical lenses, guided waves, and so on [1]. However, the time step size of the conventional BOR-FDTD algorithm is strictly limited by the Courant-Friedrichs-Lewy (CFL) condition [1]. To remove the stability limit on the time step size of the BOR-FDTD algorithm and improve the efficiency, some unconditionally stable schemes such as the alternatingdirection implicit (ADI) BOR-FDTD [3], the locally onedimensional (LOD) BOR-FDTD [4], and the weighted Laguerre polynomials (WLP) BOR-FDTD [5] algorithms have been proposed. The WLP-BOR-FDTD algorithm needs to solve a large sparse matrix, so it is not so applicable for large computational domain [5]. The LOD-BOR-FDTD algorithm and the ADI-BOR-FDTD algorithm show comparable accuracy, and the LOD-BOR-FDTD algorithm shows a little higher computational efficiency [4]. In the conventional ADIBOR-FDTD and LOD-BOR-FDTD algorithms, the equations for one full time step are split into two subtime steps; as a result, their computational expenditures are increased $[3,4]$. Recently, the one-step leapfrog ADI-FDTD algorithm which eliminates the midtime step successfully has been proposed and developed [6-11]. It makes the simulation with the ADIFDTD algorithm more efficient. The application of the onestep leapfrog ADI-FDTD algorithm to BOR has also been proposed [12]. In fact, the parallel improvement can also be made for the conventional LOD-BOR-FDTD algorithm.

Recently, an unconditionally stable one-step leapfrog LOD-FDTD algorithm was proposed [13]. In the algorithm, the equations are obtained by the manipulation of those used in the conventional LOD-FDTD algorithm. The resultant electric and magnetic field equations are interlaced half a time step apart and no subtime steps are involved. The new algorithm obtains a higher computational efficiency while preserving the properties of the conventional LOD-FDTD algorithm [13]. 
In this work, the one-step leapfrog LOD-FDTD algorithm for BOR is developed, called one-step leapfrog LOD-BORFDTD algorithm. In the proposed algorithm, the $z$-direction electric and magnetic field components are dealt with differently. Moreover, the convolutional perfectly matched layer (CPML) [14] is introduced to the one-step leapfrog LOD-BOR-FDTD algorithm. The equations of the one-step leapfrog CPML are concise.

The remainder of the paper is organized as follows. In Section 2, the equations of the one-step leapfrog LOD-BORFDTD algorithm are presented and some discussions about the algorithm are made. In Section 3, the CPML is developed for the proposed algorithm. To assess the proposed algorithm and its CPML, numerical examples are given in Section 4. Finally, conclusions are made in Section 5.

\section{Formulations and Discussions}

2.1. Equations for Off-Axis Cells. The equations for the conventional LOD-BOR-FDTD algorithm can be expressed as [4]

$$
\begin{aligned}
& \mathbf{E}^{n+1 / 2}-\frac{\Delta t}{2 \varepsilon} \mathbf{A}_{12} \mathbf{H}^{n+1 / 2}=\mathbf{E}^{n}+\frac{\Delta t}{2 \varepsilon} \mathbf{A}_{12} \mathbf{H}^{n} \\
& \mathbf{H}^{n+1 / 2}-\frac{\Delta t}{2 \mu} \mathbf{A}_{21} \mathbf{E}^{n+1 / 2}=\mathbf{H}^{n}+\frac{\Delta t}{2 \mu} \mathbf{A}_{21} \mathbf{E}^{n}
\end{aligned}
$$

for the first subtime step and

$$
\begin{aligned}
& \mathbf{E}^{n+1}+\frac{\Delta t}{2 \varepsilon} \mathbf{B}_{12} \mathbf{H}^{n+1}=\mathbf{E}^{n+1 / 2}-\frac{\Delta t}{2 \varepsilon} \mathbf{B}_{12} \mathbf{H}^{n+1 / 2} \\
& \mathbf{H}^{n+1}+\frac{\Delta t}{2 \mu} \mathbf{B}_{21} \mathbf{E}^{n+1}=\mathbf{H}^{n+1 / 2}-\frac{\Delta t}{2 \mu} \mathbf{B}_{21} \mathbf{E}^{n+1 / 2}
\end{aligned}
$$

for the second subtime step.

Here,

$$
\begin{aligned}
\mathbf{A}_{12}= & {\left[\begin{array}{ccc}
0 & 0 & \pm \frac{m}{\rho} \\
\frac{\partial}{\partial z} & 0 & 0 \\
0 & \frac{\partial(\rho)}{\rho \partial \rho} & 0
\end{array}\right], } \\
\mathbf{A}_{21}= & {\left[\begin{array}{ccc}
0 & \frac{\partial}{\partial z} & 0 \\
0 & 0 & \frac{\partial}{\partial \rho} \\
\mp \frac{m}{\rho} & 0 & 0
\end{array}\right] } \\
\mathbf{B}_{12}= & {\left[\begin{array}{ccc}
0 & \frac{\partial}{\partial z} & 0 \\
0 & 0 & \frac{\partial}{\partial \rho} \\
\pm \frac{m}{\rho} & 0 & 0
\end{array}\right] }
\end{aligned}
$$

$$
\mathbf{B}_{21}=\left[\begin{array}{ccc}
0 & 0 & \mp \frac{m}{\rho} \\
\frac{\partial}{\partial z} & 0 & 0 \\
0 & \frac{\partial(\rho)}{\rho \partial \rho} & 0
\end{array}\right]
$$

are the matrices that contain the spatial differential operators, $\mathbf{E}=\left[\begin{array}{lll}E_{\rho} & E_{\phi} & E_{z}\end{array}\right]^{T}$ and $\mathbf{H}=\left[\begin{array}{lll}H_{\rho} & H_{\phi} & H_{z}\end{array}\right]^{T}$ are the electric and magnetic field vectors, $m$ is the mode number, $\varepsilon$ is the permittivity, and $\mu$ is the permeability.

Following the similar procedure used in $[13,15,16]$, one can obtain the following electric field equations for the onestep leapfrog LOD-BOR-FDTD algorithm with only algebraic manipulations of (1a), (1b), (2a), and (2b):

$$
\begin{aligned}
\widetilde{\mathbf{E}}^{n-1 / 2} & =\mathbf{E}^{n-1 / 2}-\frac{\Delta t}{2 \varepsilon} \mathbf{B}_{12} \mathbf{h}^{n} \\
\left(\frac{\mathbf{I}}{2}-\frac{\Delta t^{2}}{8 \varepsilon \mu} \mathbf{A}_{12} \mathbf{A}_{21}\right) \mathbf{e}^{n+1 / 2} & =\widetilde{\mathbf{E}}^{n-1 / 2}+\frac{\Delta t}{2 \varepsilon} \mathbf{A}_{12} \mathbf{H}^{n} \\
\mathbf{E}^{n+1 / 2} & =\mathbf{e}^{n+1 / 2}-\widetilde{\mathbf{E}}^{n-1 / 2},
\end{aligned}
$$

where $\mathbf{I}$ is a $3 \times 3$ unit matrix, $\widetilde{\mathbf{E}}^{n-1 / 2}$ and $\mathbf{e}^{n+1 / 2}$ are auxiliary variables, and $\mathbf{e}^{n+1 / 2}=\mathbf{E}^{n+1 / 2}+\mathbf{E}^{n}$.

Similarly, one can obtain the equations for the magnetic fields

$$
\begin{aligned}
\widetilde{\mathbf{H}}^{n} & =\mathbf{H}^{n}+\frac{\Delta t}{2 \mu} \mathbf{A}_{21} \mathbf{e}^{n+1 / 2} \\
\left(\frac{\mathbf{I}}{2}-\frac{\Delta t^{2}}{8 \varepsilon \mu} \mathbf{B}_{21} \mathbf{B}_{12}\right) \mathbf{h}^{n+1} & =\widetilde{\mathbf{H}}^{n}-\frac{\Delta t}{2 \mu} \mathbf{B}_{21} \mathbf{E}^{n+1 / 2} \\
\mathbf{H}^{n+1} & =\mathbf{h}^{n+1}-\widetilde{\mathbf{H}}^{n},
\end{aligned}
$$

where $\widetilde{\mathbf{H}}^{n}$ and $\mathbf{h}^{n+1}$ are auxiliary variables and $\mathbf{h}^{n+1}=\mathbf{H}^{n+1}+$ $\mathbf{H}^{n+1 / 2}$.

2.2. Equations for On-Axis $E_{z}$ Field. The on-axis $E_{z}$ field component cannot be obtained by using (4a), (4b), and (4c) directly. Note that the on-axis $E_{z}$ field component is zero for $m \neq 0$, so one should only deal with it for $m=0$. The equation for the on-axis $E_{z}$ field component in the conventional LODBOR-FDTD algorithm is [4]

$$
\begin{gathered}
E_{z}^{n+1 / 2}\left(0, k+\frac{1}{2}\right)-\frac{2 \Delta t}{\varepsilon \Delta \rho} H_{\phi}^{n+1 / 2}\left(\frac{1}{2}, k+\frac{1}{2}\right) \\
=E_{z}^{n}\left(0, k+\frac{1}{2}\right)+\frac{2 \Delta t}{\varepsilon \Delta \rho} H_{\phi}^{n}\left(\frac{1}{2}, k+\frac{1}{2}\right)
\end{gathered}
$$

for the first subtime step and

$$
E_{z}^{n+1}\left(0, k+\frac{1}{2}\right)=E_{z}^{n+1 / 2}\left(0, k+\frac{1}{2}\right)
$$


for the second subtime step. Moreover, one can obtain the equation for $H_{\phi}^{n+1 / 2}(1 / 2, k+1 / 2)$ in the conventional LODBOR-FDTD algorithm from (1b)

$$
\begin{aligned}
H_{\phi}^{n+1 / 2} & \left(\frac{1}{2}, k+\frac{1}{2}\right) \\
& -\frac{\Delta t}{2 \mu} \frac{E_{z}^{n+1 / 2}(1, k+1 / 2)-E_{z}^{n+1 / 2}(0, k+1 / 2)}{\Delta \rho} \\
= & H_{\phi}^{n}\left(\frac{1}{2}, k+\frac{1}{2}\right) \\
& +\frac{\Delta t}{2 \mu} \frac{E_{z}^{n}(1, k+1 / 2)-E_{z}^{n}(0, k+1 / 2)}{\Delta \rho} .
\end{aligned}
$$

Applying the similar procedure used in Section 2.1, one can obtain the following equations for the on-axis $E_{z}$ field component by using (6a), (6b), and (7):

$$
\begin{aligned}
& \left(\frac{1}{2}+\frac{\Delta t^{2}}{2 \varepsilon \mu \Delta \rho^{2}}\right) e_{z}^{n+1 / 2}\left(0, k+\frac{1}{2}\right) \\
& \quad-\frac{\Delta t^{2}}{2 \varepsilon \mu \Delta \rho^{2}} e_{z}^{n+1 / 2}\left(1, k+\frac{1}{2}\right)=E_{z}^{n-1 / 2}\left(0, k+\frac{1}{2}\right) \\
& \quad+\frac{2 \Delta t}{\varepsilon \Delta \rho} H_{\phi}^{n}\left(\frac{1}{2}, k+\frac{1}{2}\right) \\
& E_{z}^{n+1 / 2}\left(0, k+\frac{1}{2}\right)=e_{z}^{n+1 / 2}\left(0, k+\frac{1}{2}\right) \\
& \quad-E_{z}^{n-1 / 2}\left(0, k+\frac{1}{2}\right) .
\end{aligned}
$$

Unlike the equations for the off-axis $E_{z}$ field components, no auxiliary variable $\widetilde{E}_{z}$ is involved here.

2.3. Equations for $H_{z}^{n+1}(1 / 2, k)$. The equations for $H_{z}^{n+1}(1 / 2, k)$ should also be treated specially. With the algebraic manipulation of the relative difference equations used in the conventional LOD-BOR-FDTD algorithm [4], one can obtain the following equations for $H_{z}^{n+1}(1 / 2, k)$ :

$$
\begin{gathered}
\widetilde{H}_{z}^{n}\left(\frac{1}{2}, k\right)=H_{z}^{n}\left(\frac{1}{2}, k\right)+\frac{\Delta t}{\mu}\left(\mp \frac{m}{\Delta \rho}\right) e_{\rho}^{n+1 / 2}\left(\frac{1}{2}, k\right) \\
\left(\frac{1}{2}+\frac{\Delta t^{2}}{4 \varepsilon \mu \Delta \rho^{2}}\right) h_{z}^{n+1}\left(\frac{1}{2}, k\right)-\frac{\Delta t^{2}}{4 \varepsilon \mu \Delta \rho^{2}} h_{z}^{n+1}\left(\frac{3}{2}, k\right) \\
=\widetilde{H}_{z}^{n}\left(\frac{1}{2}, k\right)-\frac{\Delta t}{\mu \Delta \rho} E_{\phi}^{n+1 / 2}(1, k) \\
H_{z}^{n+1}\left(\frac{1}{2}, k\right)=h_{z}^{n+1}\left(\frac{1}{2}, k\right)-\widetilde{H}_{z}^{n}\left(\frac{1}{2}, k\right) .
\end{gathered}
$$

2.4. Some Discussions about the Proposed Algorithm. It can be seen that all the equations used in the proposed algorithm are obtained from those used in the conventional LOD-BORFDTD algorithm and only algebraic manipulations are made. Therefore, one can conclude that the proposed algorithm preserves the properties of the conventional LOD-BORFDTD algorithm.

In terms of memory, the variables used in the conventional LOD-BOR-FDTD algorithm are $\mathbf{E}^{n+1 / 2}, \mathbf{H}^{n+1 / 2}$, and $\mathbf{E}^{n}$ in the first subtime step, which can also be reused in the second subtime step. In the proposed algorithm, $\widetilde{\mathbf{E}}^{n-1 / 2}$, $\mathbf{E}^{n-1 / 2}$, and $\mathbf{E}^{n+1 / 2}$ can occupy the same memory space. There are similar situations to $\widetilde{\mathbf{H}}^{n}, \mathbf{H}^{n}$, and $\mathbf{H}^{n+1}$ and $\mathbf{e}^{n+1 / 2}$ and $\mathbf{h}^{n+1}$. As a result, the proposed algorithm consumes the same amount of memory as the conventional LOD-BOR-FDTD algorithm.

There are four tridiagonal equations in one full time step, for both the conventional LOD-BOR-FDTD algorithm and the proposed algorithm. So the floating point operations at the left-hand sides of the equations are the same for the two algorithms. However, the numbers of the multiplications/divisions (M/D) and the additions/subtractions (A/S) at the right-hand sides of the equations are different. Table 1 shows the count of the $\mathrm{M} / \mathrm{D}$ and $\mathrm{A} / \mathrm{S}$ at the right-hand sides of the equations in two algorithms for modes $m=0$ and $m \neq 0$, respectively. Obviously, the proposed algorithm needs less floating point operations, so one can conclude that it has a higher computational efficiency. Note that all the coefficients of the equations are precomputed and stored here, and special treatments for the components that lie on or near the axis are not considered.

In the proposed algorithm, tridiagonal implicit equations for the magnetic field components are involved, so one should pay special attention to handling the perfectly electrically conducting (PEC) boundary, since there will be out-ofdomain magnetic field components. To address this issue, one can resort to the image theory [17]. Such treatments are often used in handling the PEC resonator, PEC scatterer, PEC wall of the perfectly matched layer (PML), and so on.

\section{CPML Implementation for the Proposed Algorithm}

To solve open region problems efficiently, the CPML for the proposed one-step leapfrog LOD-BOR-FDTD algorithm is developed. The coordinate-stretching variables for the CPML are defined as

$$
\begin{aligned}
& s_{\rho}=\kappa_{\rho}+\frac{\sigma_{\rho}}{\alpha_{\rho}+j \omega \varepsilon}, \\
& s_{z}=\kappa_{z}+\frac{\sigma_{z}}{\alpha_{z}+j \omega \varepsilon} .
\end{aligned}
$$

One can also define

$$
s_{\phi}=\frac{\tilde{\rho}}{\rho}=\kappa_{\phi}+\frac{\sigma_{\phi}}{\alpha_{\phi}+j \omega \varepsilon},
$$

where $s_{\phi}=(1 / \rho)\left[\rho_{0}+\int_{\rho_{0}}^{\rho}\left(\kappa_{\rho}+\left(\sigma_{\rho} /\left(\alpha_{\rho}+j \omega \varepsilon\right)\right)\right) d \rho^{\prime}\right]$ and $\rho_{0}$ represents the interface between FDTD and PML domains [18].

For simplicity, the CPML equations for the $E_{z}$ field component are given here. In fact, the CPML equations for 
TABLE 1: The floating point operations at the right-hand sides of the equations for two algorithms.

\begin{tabular}{lccc}
\hline Mode & & Conventional LOD-BOR-FDTD & \multicolumn{2}{c}{ One-step leapfrog } \\
LOD-BOR-FDTD \\
& $m=0$ & $m \neq 0$ & $m=0$ \\
\hline M/D & 20 & 26 & 12 \\
A/S & 32 & 38 & 22 \\
M/D + A/S & 52 & 64 & 26 \\
\hline
\end{tabular}

the other field components can be obtained in a similar manner. According to the definition in (10) and (11), one can write the CPML equations of the conventional LOD-BORFDTD algorithm in the following form:

$$
\begin{gathered}
E_{z}^{n+1 / 2}-\frac{\Delta t}{2 \varepsilon}\left(\frac{1}{\kappa_{\phi}} \frac{H_{\phi}^{n+1 / 2}}{\rho}+\frac{1}{\kappa_{\rho}} D_{\rho} H_{\phi}^{n+1 / 2}+\psi_{e z \rho \phi}^{n+1 / 2}\right. \\
\left.+\psi_{e z \rho}^{n+1 / 2}\right)=E_{z}^{n}+\frac{\Delta t}{2 \varepsilon}\left(\frac{1}{\kappa_{\phi}} \frac{H_{\phi}^{n}}{\rho}+\frac{1}{\kappa_{\rho}} D_{\rho} H_{\phi}^{n}\right. \\
\left.+\psi_{e z \rho \phi}^{n}+\psi_{e z \rho}^{n}\right) \\
H_{\phi}^{n+1 / 2}-\frac{\Delta t}{2 \mu}\left(\frac{1}{\kappa_{\rho}} D_{\rho} E_{z}^{n+1 / 2}+\psi_{h \phi \rho}^{n+1 / 2}\right)=H_{\phi}^{n} \\
+\frac{\Delta t}{2 \mu}\left(\frac{1}{\kappa_{\rho}} D_{\rho} E_{z}^{n}+\psi_{h \phi \rho}^{n}\right)
\end{gathered}
$$

for the first subtime step and

$$
\begin{aligned}
& E_{z}^{n+1}+\frac{\Delta t( \pm m)}{2 \varepsilon}\left(\frac{1}{\kappa_{\phi}} \frac{H_{\rho}^{n+1}}{\rho}+\psi_{e z \phi}^{n+1}\right) \\
& =E_{z}^{n+1 / 2}-\frac{\Delta t( \pm m)}{2 \varepsilon}\left(\frac{1}{\kappa_{\phi}} \frac{H_{\rho}^{n+1 / 2}}{\rho}+\psi_{e z \phi}^{n+1 / 2}\right) \\
& H_{\phi}^{n+1}+\frac{\Delta t}{2 \mu}\left(\frac{1}{\kappa_{z}} D_{z} E_{\rho}^{n+1}+\psi_{h \phi z}^{n+1}\right) \\
& =H_{\phi}^{n+1 / 2}-\frac{\Delta t}{2 \mu}\left(\frac{1}{\kappa_{z}} D_{z} E_{\rho}^{n+1 / 2}+\psi_{h \phi z}^{n+1 / 2}\right)
\end{aligned}
$$

for the second subtime step.

In the first subtime step, one can define

$$
\begin{gathered}
e_{z}^{n+1 / 2}=E_{z}^{n+1 / 2}+E_{z}^{n} \\
h_{\phi}^{n+1 / 2}=H_{\phi}^{n+1 / 2}+H_{\phi}^{n} \\
\varphi_{e z \rho \phi}^{n+1 / 2}=\psi_{e z \rho \phi}^{n+1 / 2}+\psi_{e z \rho \phi}^{n} \\
\varphi_{e z \rho}^{n+1 / 2}=\psi_{e z \rho}^{n+1 / 2}+\psi_{e z \rho}^{n} \\
\varphi_{h \phi \rho}^{n+1 / 2}=\psi_{h \phi \rho}^{n+1 / 2}+\psi_{h \phi \rho}^{n} .
\end{gathered}
$$

Then (12a) and (12b) can be rewritten as

$$
\begin{gathered}
e_{z}^{n+1 / 2}-\frac{\Delta t}{2 \varepsilon}\left(\frac{1}{\kappa_{\phi}} \frac{h_{\phi}^{n+1 / 2}}{\rho}+\frac{1}{\kappa_{\rho}} D_{\rho} h_{\phi}^{n+1 / 2}+\varphi_{e z \rho \phi}^{n+1 / 2}\right. \\
\left.+\varphi_{\text {ez }}^{n+1 / 2}\right)=2 E_{z}^{n} \\
h_{\phi}^{n+1 / 2}-\frac{\Delta t}{2 \mu}\left(\frac{1}{\kappa_{\rho}} D_{\rho} e_{z}^{n+1 / 2}+\varphi_{h \phi \rho}^{n+1 / 2}\right)=2 H_{\phi}^{n} .
\end{gathered}
$$

In the second subtime step, one can define

$$
\begin{aligned}
& e_{z}^{n+1}=E_{z}^{n+1}+E_{z}^{n+1 / 2} \\
& h_{\rho}^{n+1}=H_{\rho}^{n+1}+H_{\rho}^{n+1 / 2} \\
& \varphi_{e z \phi}^{n+1}=\psi_{e z \phi}^{n+1}+\psi_{e z \phi}^{n+1 / 2} .
\end{aligned}
$$

Then (13a) can be rewritten as

$$
e_{z}^{n+1}+\frac{\Delta t( \pm m)}{2 \varepsilon}\left(\frac{1}{\kappa_{\phi}} \frac{h_{\rho}^{n+1}}{\rho}+\varphi_{e z \phi}^{n+1}\right)=2 E_{z}^{n+1 / 2}
$$

The former time steps of (16a) and (17) are

$$
\begin{aligned}
e_{z}^{n} & =E_{z}^{n}+E_{z}^{n-1 / 2} \\
e_{z}^{n}+\frac{\Delta t( \pm m)}{2 \varepsilon}\left(\frac{1}{\kappa_{\phi}} \frac{h_{\rho}^{n}}{\rho}+\varphi_{e z \phi}^{n}\right) & =2 E_{z}^{n-1 / 2}
\end{aligned}
$$

Substituting (19) into (18), one can obtain

$$
E_{z}^{n-1 / 2}-\frac{\Delta t( \pm m)}{2 \varepsilon}\left(\frac{1}{\kappa_{\phi}} \frac{h_{\rho}^{n}}{\rho}+\varphi_{e z \phi}^{n}\right)=E_{z}^{n} .
$$


Substituting (15b) into (15a), one can obtain

$$
\begin{aligned}
e_{z}^{n+1 / 2} & -\frac{\Delta t}{\varepsilon} \frac{1}{\kappa_{\phi}} \frac{1}{\rho} H_{\phi}^{n} \\
& -\frac{\Delta t}{2 \varepsilon} \frac{1}{\kappa_{\phi}} \frac{1}{\rho} \frac{\Delta t}{2 \mu}\left(\frac{1}{\kappa_{\rho}} D_{\rho} e_{z}^{n+1 / 2}+\varphi_{h \phi \rho}^{n+1 / 2}\right) \\
& -\frac{\Delta t}{\varepsilon} \frac{1}{\kappa_{\rho}} D_{\rho} H_{\phi}^{n} \\
& -\frac{\Delta t}{2 \varepsilon} \frac{1}{\kappa_{\rho}} D_{\rho} \frac{\Delta t}{2 \mu}\left(\frac{1}{\kappa_{\rho}} D_{\rho} e_{z}^{n+1 / 2}+\varphi_{h \phi \rho}^{n+1 / 2}\right) \\
- & \frac{\Delta t}{2 \varepsilon}\left(\varphi_{e z \rho \phi}^{n+1 / 2}+\varphi_{e z \rho}^{n+1 / 2}\right)=2 E_{z}^{n} .
\end{aligned}
$$

Substituting (20) into (21), the following equation can be obtained:

$$
\begin{aligned}
e_{z}^{n+1 / 2} & -\frac{\Delta t}{2 \varepsilon} \frac{1}{\kappa_{\phi}} \frac{1}{\rho} \frac{\Delta t}{2 \mu}\left(\frac{1}{\kappa_{\rho}} D_{\rho} e_{z}^{n+1 / 2}+\varphi_{h \phi \rho}^{n+1 / 2}\right) \\
& -\frac{\Delta t}{2 \varepsilon} \frac{1}{\kappa_{\rho}} D_{\rho} \frac{\Delta t}{2 \mu}\left(\frac{1}{\kappa_{\rho}} D_{\rho} e_{z}^{n+1 / 2}+\varphi_{h \phi \rho}^{n+1 / 2}\right) \\
= & 2 E_{z}^{n-1 / 2}-\frac{\Delta t( \pm m)}{\varepsilon}\left(\frac{1}{\kappa_{\phi}} \frac{h_{\rho}^{n}}{\rho}+\varphi_{e z \phi}^{n}\right) \\
& +\frac{\Delta t}{\varepsilon} \frac{1}{\kappa_{\phi}} \frac{1}{\rho} H_{\phi}^{n}+\frac{\Delta t}{\varepsilon} \frac{1}{\kappa_{\rho}} D_{\rho} H_{\phi}^{n} \\
+ & \frac{\Delta t}{2 \varepsilon}\left(\varphi_{e z \rho \phi}^{n+1 / 2}+\varphi_{e z \rho}^{n+1 / 2}\right) .
\end{aligned}
$$

Some of the auxiliary variables in (22) are approximated as

$$
\begin{aligned}
& \varphi_{h \phi \rho}^{n+1 / 2}=\varphi_{h \phi \rho}^{n-1 / 2}+O(\Delta t) \\
& \varphi_{e z \rho \phi}^{n+1 / 2}=\psi_{e z \rho \phi}^{n+1 / 2}+\psi_{e z \rho \phi}^{n}=2 \psi_{e z \rho \phi}^{n}+O(\Delta t) \\
& \varphi_{e z \rho}^{n+1 / 2}=\psi_{e z \rho}^{n+1 / 2}+\psi_{e z \rho}^{n}=2 \psi_{e z \rho}^{n}+O(\Delta t) .
\end{aligned}
$$

Ignoring the high order terms of $\Delta t$ in (23a), (23b), and (23c), then (22) can be approximated as

$$
\begin{aligned}
e_{z}^{n+1 / 2} & -\frac{\Delta t}{2 \varepsilon} \frac{1}{\kappa_{\phi}} \frac{1}{\rho} \frac{\Delta t}{2 \mu} \frac{1}{\kappa_{\rho}} D_{\rho} e_{z}^{n+1 / 2} \\
& -\frac{\Delta t}{2 \varepsilon} \frac{1}{\kappa_{\rho}} D_{\rho} \frac{\Delta t}{2 \mu} \frac{1}{\kappa_{\rho}} D_{\rho} e_{z}^{n+1 / 2} \\
= & 2 E_{z}^{n-1 / 2}-\frac{\Delta t( \pm m)}{\varepsilon}\left(\frac{1}{\kappa_{\phi}} \frac{h_{\rho}^{n}}{\rho}+\varphi_{e z \phi}^{n}\right) \\
& +\frac{\Delta t}{\varepsilon}\left(\frac{1}{\kappa_{\phi}} \frac{1}{\rho} H_{\phi}^{n}+\psi_{e z \rho \phi}^{n}\right)
\end{aligned}
$$

$$
\begin{aligned}
& +\frac{\Delta t}{\varepsilon}\left(\frac{1}{\kappa_{\rho}} D_{\rho} H_{\phi}^{n}+\psi_{e z \rho}^{n}\right)+\frac{\Delta t}{2 \varepsilon} \frac{1}{\kappa_{\phi}} \frac{1}{\rho} \frac{\Delta t}{2 \mu} \varphi_{h \phi \rho}^{n-1 / 2} \\
& +\frac{\Delta t}{2 \varepsilon} \frac{1}{\kappa_{\rho}} D_{\rho} \frac{\Delta t}{2 \mu} \varphi_{h \phi \rho}^{n-1 / 2} .
\end{aligned}
$$

The following equation can be obtained from (14a) and (20):

$$
E_{z}^{n+1 / 2}=e_{z}^{n+1 / 2}-E_{z}^{n-1 / 2}+\frac{\Delta t( \pm m)}{2 \varepsilon}\left(\frac{1}{\kappa_{\phi}} \frac{h_{\rho}^{n}}{\rho}+\varphi_{e z \phi}^{n}\right) .
$$

To simplify the equations further, one can introduce an auxiliary variable $\widetilde{E}_{z}^{n-1 / 2}$. As a result, the final equations for $E_{z}$ can be reformulated as

$$
\begin{aligned}
& \widetilde{E}_{z}^{n-1 / 2}=E_{z}^{n-1 / 2}-\frac{\Delta t( \pm m)}{2 \varepsilon}\left(\frac{1}{\kappa_{\phi}} \frac{h_{\rho}^{n}}{\rho}+\varphi_{e z \phi}^{n}\right) \\
& \frac{1}{2} e_{z}^{n+1 / 2}-\frac{\Delta t^{2}}{8 \varepsilon \mu} \frac{1}{\kappa_{\phi}} \frac{1}{\rho} \frac{1}{\kappa_{\rho}} D_{\rho} e_{z}^{n+1 / 2} \\
& -\frac{\Delta t^{2}}{8 \varepsilon \mu} \frac{1}{\kappa_{\rho}} D_{\rho} \frac{1}{\kappa_{\rho}} D_{\rho} e_{z}^{n+1 / 2}=\widetilde{E}_{z}^{n-1 / 2} \\
& +\frac{\Delta t}{2 \varepsilon}\left(\frac{1}{\kappa_{\phi}} \frac{1}{\rho} H_{\phi}^{n}+\psi_{e z \rho \phi}^{n}\right) \\
& +\frac{\Delta t}{2 \varepsilon}\left(\frac{1}{\kappa_{\rho}} D_{\rho} H_{\phi}^{n}+\psi_{e z \rho}^{n}\right)+\frac{\Delta t^{2}}{8 \varepsilon \mu} \frac{1}{\kappa_{\phi}} \frac{1}{\rho} \varphi_{h \phi \rho}^{n-1 / 2} \\
& +\frac{\Delta t^{2}}{8 \varepsilon \mu} \frac{1}{\kappa_{\rho}} D_{\rho} \varphi_{h \phi \rho}^{n-1 / 2} \\
& E_{z}^{n+1 / 2}=e_{z}^{n+1 / 2}-\widetilde{E}_{z}^{n-1 / 2} .
\end{aligned}
$$

Some of the auxiliary variables in (26a), (26b), and (26c) can be solved by

$$
\begin{aligned}
& \varphi_{e z \phi}^{n}=b_{\phi} \varphi_{e z \phi}^{n-1}+a_{\phi}\left(\frac{h_{\rho}}{\rho}\right)^{n} \\
& \psi_{e z \rho \phi}^{n}=b_{\phi} \psi_{e z \rho \phi}^{n-1}+a_{\phi}\left(\frac{H_{\phi}}{\rho}\right)^{n} \\
& \psi_{e z \rho}^{n}=b_{\rho} \psi_{e z \rho}^{n-1}+a_{\rho}\left(\frac{\partial H_{\phi}}{\partial \rho}\right)^{n}
\end{aligned}
$$

where $b_{\varsigma}=e^{-\left(\sigma_{\zeta} / \kappa_{\varsigma}+\alpha_{\zeta}\right) \Delta t / \varepsilon}$ and $a_{\varsigma}=\left(\sigma_{\varsigma} /\left(\kappa_{\varsigma}\left(\sigma_{\zeta}+\kappa_{\varsigma} \alpha_{\varsigma}\right)\right)\right)\left(b_{\varsigma}-1\right)$, $\varsigma=\rho, \phi, z$.

In addition, the auxiliary variable $\varphi_{h \phi \rho}^{n-1 / 2}$ in (26b) has been solved in updating $H_{\phi}^{n}$, so it can be used directly here. It is obvious that the number of the convolutional auxiliary variables for the proposed CPML is the same as that of the CPML for the conventional BOR-FDTD algorithm. 
TABLE 2: Comparison of the computational results with different algorithms.

\begin{tabular}{lcccccccccc}
\hline & \multirow{2}{*}{ BOR-FDTD } & \multicolumn{2}{c}{ Conventional LOD-BOR-FDTD } & \multicolumn{3}{c}{$\begin{array}{c}\text { One-step leapfrog } \\
\text { ADI-BOR-FDTD }\end{array}$} & \multicolumn{3}{c}{$\begin{array}{c}\text { One-step leapfrog } \\
\text { LOD-BOR-FDTD }\end{array}$} \\
\hline CFLN & 1 & 1 & 4 & 8 & 1 & 4 & 8 & 1 & 4 & 8 \\
Iterations & 40000 & 40000 & 10000 & 5000 & 40000 & 10000 & 5000 & 40000 & 10000 & 5000 \\
Results, GHz & 4.954 & 4.954 & 4.943 & 4.907 & 4.954 & 4.943 & 4.907 & 4.954 & 4.943 & 4.907 \\
Relative error & $0.08 \%$ & $0.08 \%$ & $-0.14 \%$ & $-0.87 \%$ & $0.08 \%$ & $-0.14 \%$ & $-0.87 \%$ & $0.08 \%$ & $-0.14 \%$ & $-0.87 \%$ \\
Time, s & 13.2 & 52.8 & 13.2 & 6.4 & 43.2 & 10.9 & 5.5 & 38.8 & 9.8 & 4.8 \\
\hline
\end{tabular}

Consequently, one can say that the equations of proposed CPML are concise.

When $\kappa=1$ and $\sigma=0$, the equations of the proposed CPML can be simplified to be the normal equations of the proposed LOD-BOR-FDTD algorithm. Similarly, since the coordinate stretching along the $\rho$-direction only exists in the radial outer boundary, that is, $\kappa_{\rho}=\kappa_{\phi}=1$ and $\sigma_{\rho}=\sigma_{\phi}=0$ on (or near) the axis, the CPML equations for $E_{z}^{n+1 / 2}(0, k+1 / 2)$ and $H_{z}^{n+1}(1 / 2, k)$ are still (8a), (8b), (9a), (9b), and (9c).

In the Cartesian coordinate system, the coordinate stretching along each axis is similar to that along the $z$ direction in the cylindrical coordinate system. So it can be concluded that the CPML for the LOD-FDTD algorithm in the Cartesian coordinate system [13] can be implemented by applying the similar procedure.

\section{Numerical Results and Discussions}

A resonant cavity and a scattering example are provided to validate the one-step leapfrog LOD-BOR-FDTD algorithm and its CPML.

4.1. Cavity Resonator. The $\mathrm{TE}_{011}$ mode of a PEC cylindrical cavity resonator is computed, which is frequently used as a benchmark $[3,4]$. The radius and height of the cavity are $3.995 \mathrm{~cm}$ and $7.910 \mathrm{~cm}$, respectively. Its theoretical resonant frequency is $4.950 \mathrm{GHz}[3,4]$. The computational domain is divided into $50(\rho) \times 100(z)$ cells, with $\Delta \rho=0.0799 \mathrm{~cm}$ and $\Delta z=0.0791 \mathrm{~cm}$. The cavity is computed with the conventional BOR-FDTD algorithm, the conventional LODBOR-FDTD algorithm, the one-step leapfrog ADI-BORFDTD algorithm, and the proposed algorithm, respectively. A time history of $52.73 \mathrm{~ns}$ is simulated.

Table 2 shows the computational results with different algorithms. Note that CFLN $=\Delta t / \Delta t_{\mathrm{CFL}}$ and $\Delta t_{\mathrm{CFL}}$ is the maximum time step size that satisfies the CFL condition for the conventional BOR-FDTD algorithm [1], which is $\Delta t_{\mathrm{CFL}}=$ $1.318 \times 10^{-12} \mathrm{~s}$ here. It can be seen from Table 2 that the one-step leapfrog LOD-BOR-FDTD algorithm obtains the same numerical results as the conventional LOD-BOR-FDTD algorithm. Moreover, the numerical errors of two algorithms increase as the time step sizes increase. In terms of the computational time, the proposed algorithm consumes about $26.5 \%, 25.8 \%$, and $25.0 \%$ less time than the conventional LOD-BOR-FDTD algorithm when CFLN is chosen to be 1,4 , and 8 , respectively. The two unconditionally stable algorithms use the same amount of memory, which is $1.06 \mathrm{MB}$. Therefore, one can say that the proposed algorithm preserves the properties of the conventional LOD-BOR-FDTD algorithm and obtains a higher computational efficiency. The proposed algorithm also shows the same accuracy as that of the onestep leapfrog ADI-BOR-FDTD algorithm but obtains a little higher computational efficiency, as shown in Table 2. Note that the comparison among the conventional LOD-BORFDTD algorithm, the one-step leapfrog ADI-BOR-FDTD algorithm, and the proposed algorithm was performed only in the case of the resonant cavity and that further analysis could be performed on other situations to rule out the possibility that the results could be linked to the particular case considered. Since the $\mathrm{TE}_{011}$ mode is computed, the terms including multiplicative factor $m$ (mode number) in the equations are all zero. In the simulation, these terms are not computed. All of the calculations are performed on an Intel ${ }^{\circledR}$ Core $^{\mathrm{TM}} 2$ Quad CPU Q6600 @ 2.40 GHz machine and the Fortran codes are used.

4.2. Scattering Example. To validate the proposed CPML for the one-step leapfrog LOD-BOR-FDTD algorithm, the scattered field from a PEC cylinder is computed. The radius and height of the cylinder are $20 \mathrm{~cm}$ and $40 \mathrm{~cm}$, respectively. The oblique incident plane wave with incident angle $\theta_{i}=45^{\circ}$ is introduced by the TF/SF boundary conditions, which are placed at $\rho=25 \mathrm{~cm}$ and $z= \pm 25 \mathrm{~cm}$. The incident plane wave is parallel polarized, as shown in Figure 1. The gray domain in Figure 1 denotes the PEC cylinder. The Gaussian pulse $E_{0} \cos \left(2 \pi f_{0} t\right) e^{-4 \pi\left(\left(t-t_{0}\right) / \tau\right)^{2}}$ with $E_{0}=1000 \mathrm{~V} / \mathrm{m}, f_{0}=$ $0.3 \mathrm{GHz}, \tau=2.2 \mathrm{~ns}$, and $t_{0}=1.25 / f_{0}$ is used as the incident electric field $E_{i}$. The small computational domain is composed of $40(\rho) \times 80(z)$ cells, containing the 10 PML layers in the outer boundary, which are denoted by the oblique-linedomain as shown in Figure 1. According to the characteristic of the problem, the mode number ranges from 0 to 11 . The uniform grids are chosen to be $\Delta \rho=\Delta z=1 \mathrm{~cm}$. Therefore, the maximum time step size for the conventional BOR-FDTD [1] is $\Delta t_{\mathrm{CFL}}=2.778 \times 10^{-12} \mathrm{~s}$ in the example. The observation point is placed at grid point $Q(29,-29)$, which is near the corner of the scattered field domain.

The scattered field is computed with the conventional BOR-FDTD algorithm and the proposed algorithm, respectively, both with the CPML absorbing boundary conditions. 


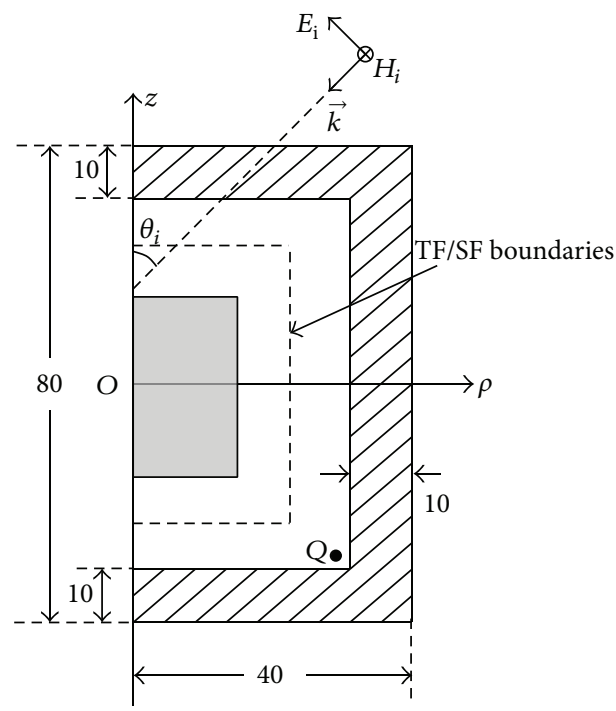

FIgURE 1: Illustration of the scattering example with oblique incidence.

The parameters of the CPML are chosen according to the following expressions [18]:

$$
\begin{aligned}
\sigma_{\varsigma} & =\frac{\sigma_{\varsigma \max }\left|\varsigma-\varsigma_{0}\right|^{p}}{\delta^{p}} \\
\sigma_{\zeta \mathrm{opt}} & =\frac{(p+1)}{150 \pi \Delta \varsigma} \\
\kappa_{\varsigma} & =1+\kappa_{\varsigma \max } \frac{\left|\varsigma-\varsigma_{0}\right|^{p}}{\delta^{p}},
\end{aligned}
$$

where $\varsigma=\rho, z, \delta$ is the thickness of the PML, $\varsigma_{0}$ is the interface between the FDTD and PML grids, and $p=4$ is the order of the polynomial. In the computing, the optimized parameters are chosen to be $\kappa_{\zeta \max }=3, \sigma_{\max }=0.4 \sigma_{\mathrm{opt}}$, and $\alpha=0.004$.

To validate the one-step leapfrog LOD-BOR-FDTD algorithm further, the scattered field is computed via large computational domain first. In this case, the scattered field at the observation point $Q$ is without reflection, so one can make a clear comparison. Figure 2 shows the $E_{z}$ fields obtained by the two algorithms via large computational domains. For the proposed algorithm, different CFLNs are used, where CFLN $=\Delta t / \Delta t_{\mathrm{CFL}}$. It can be seen that the results of the proposed algorithm agree well with those of the conventional BOR-FDTD algorithm. Therefore, the proposed algorithm is validated further. Meanwhile, one can see that the numerical error of the proposed algorithm increases as the time step size grows.

Figure 3 shows the reflection errors of the CPMLs for the conventional BOR-FDTD algorithm and the proposed algorithm, which are obtained by the $E_{z}$ fields at the observation point $Q$. The reflection error is defined as

$$
R_{\mathrm{dB}}=20 \log _{10}\left(\frac{\left|E_{\mathrm{pml}}(t)-E_{\mathrm{ref}}(t)\right|}{\max \left|E_{\mathrm{ref}}(t)\right|}\right),
$$

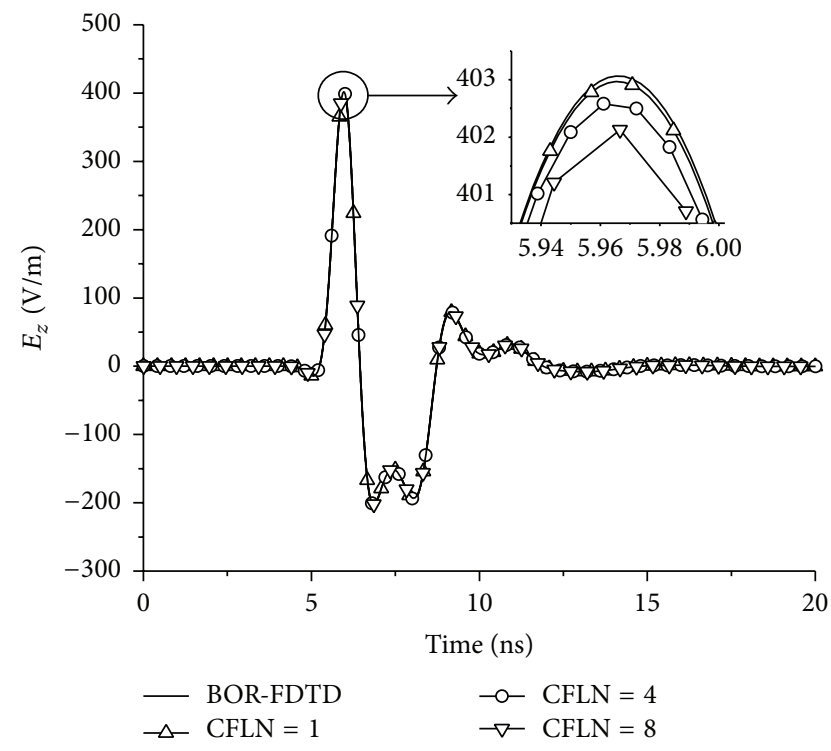

FIGURE 2: $E_{z}$ fields at the observation point $Q$ with the conventional BOR-FDTD and the proposed algorithm.

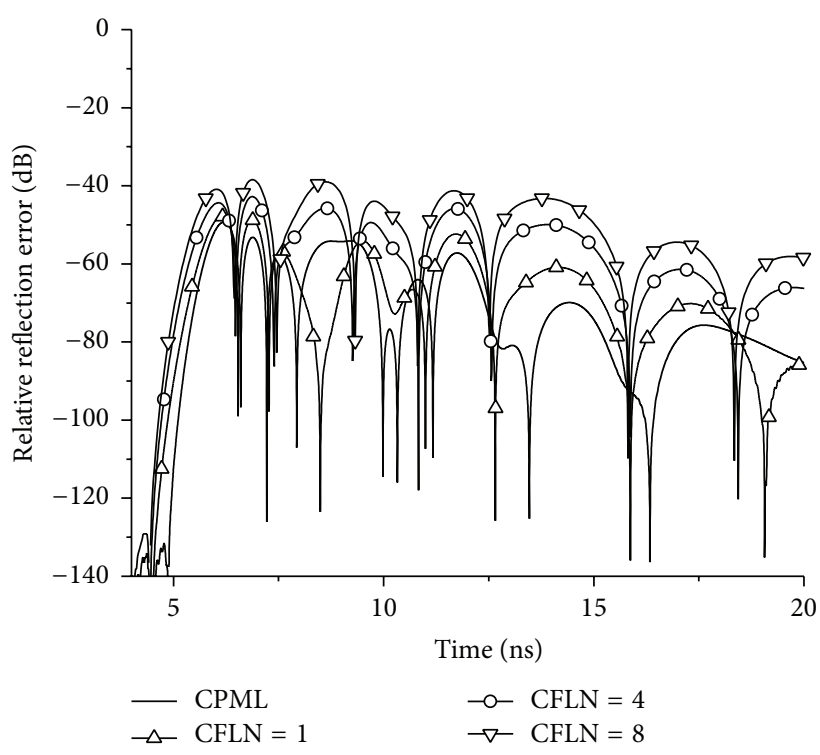

FIGURE 3: Relative reflection errors of the CPML for the conventional BOR-FDTD and the proposed CPML for the one-step leapfrog LOD-BOR-FDTD.

where $E_{\mathrm{pml}}(t)$ is the result obtained by the small computational domain as shown in Figure 1. $E_{\text {ref }}(t)$ is the numerical result via large computational domain. For the proposed algorithm, $E_{\text {ref }}(t)$ is computed separately for each CFLN. It can be seen from Figure 3 that the CPML for the one-step leapfrog LOD-BOR-FDTD algorithm shows good performance. Moreover, the reflection error of the one-step leapfrog CPML increases as the time step size grows. When CFLN $=8$, the maximum reflection error is about $-40 \mathrm{~dB}$. 


\section{Conclusion}

In this paper, the one-step leapfrog LOD-BOR-FDTD algorithm is presented. It obtains a higher computational efficiency while keeping the properties of the conventional LOD-BOR-FDTD algorithm. In addition, the CPML for the proposed algorithm is implemented. The numerical results show the performances of the one-step leapfrog LOD-BORFDTD algorithm and its CPML. It can be concluded that, by applying the similar procedure, the CPML for the LODFDTD algorithm in the Cartesian coordinate system can be implemented.

\section{Competing Interests}

The authors declare that there is no conflict of interests regarding the publication of this paper.

\section{References}

[1] A. Taflove and S. C. Hagness, Computational Electrodynamics: The Finite-difference Time-domain Method, Artech House, Boston, Mass, USA, 2nd edition, 2000.

[2] Y. Chen, R. Mittra, and P. Harms, "Finite-difference timedomain algorithm for solving maxwell's equations in rotationally symmetric geometries," IEEE Transactions on Microwave Theory and Techniques, vol. 44, no. 6, pp. 832-839, 1996.

[3] H.-L. Chen, B. Chen, Y. Yi, and D.-G. Fang, "Unconditionally stable ADI-BOR-FDTD algorithm for the analysis of rotationally symmetric geometries," IEEE Microwave and Wireless Components Letters, vol. 17, no. 4, pp. 304-306, 2007.

[4] J. Shibayama, B. Murakami, J. Yamauchi, and H. Nakano, "LODBOR-FDTD algorithm for efficient analysis of circularly symmetric structures," IEEE Microwave and Wireless Components Letters, vol. 19, no. 2, pp. 56-58, 2009.

[5] H.-L. Chen, B. Chen, Y.-T. Duan, Y. Yi, and D.-G. Fang, "Unconditionally stable laguerre-based BOR-FDTD scheme for scattering from bodies of revolution," Microwave and Optical Technology Letters, vol. 49, no. 8, pp. 1897-1900, 2007.

[6] S. J. Cooke, M. Botton, T. M. Antonsen Jr., and B. Levush, "A leapfrog formulation of the 3D ADI-FDTD algorithm," in Proceedings of the Workshop on Computational Electromagnetics in Time-Domain (CEM-TD '07), pp. 144-147, Perugia, Italy, October 2007.

[7] S.-C. Yang, Z. Chen, Y. Yu, and W.-Y. Yin, "An unconditionally stable one-step arbitrary-order leapfrog ADI-FDTD method and its numerical properties," IEEE Transactions on Antennas and Propagation, vol. 60, no. 4, pp. 1995-2003, 2012.

[8] S.-C. Yang, Z. Chen, Y.-Q. Yu, and W.-Y. Yin, "The unconditionally stable one-step leapfrog ADI-FDTD method and its comparisons with other FDTD methods," IEEE Microwave and Wireless Components Letters, vol. 21, no. 12, pp. 640-642, 2011.

[9] X.-H. Wang, W.-Y. Yin, Y.-Q. Yu, Z. D. Chen, J. Wang, and Y. Guo, "A convolutional perfect matched layer (CPML) for onestep leapfrog ADI-FDTD method and its applications to EMC problems," IEEE Transactions on Electromagnetic Compatibility, vol. 54, no. 5, pp. 1066-1076, 2012.

[10] T. H. Gan and E. L. Tan, "Analysis of the divergence properties for the three-dimensional leapfrog ADI-FDTD method," IEEE Transactions on Antennas and Propagation, vol. 60, no. 12, pp. 5801-5808, 2012.
[11] J.-Y. Gao and H.-X. Zheng, "One-step leapfrog ADI-FDTD method for lossy media and its stability analysis," Progress in Electromagnetics Research Letters, vol. 40, pp. 49-60, 2013.

[12] Y.-G. Wang, B. Chen, H.-L. Chen, and R. Xiong, "An unconditionally stable one-step leapfrog ADI-BOR-FDTD method," IEEE Antennas and Wireless Propagation Letters, vol. 12, pp. 647-650, 2013.

[13] J. Wang, B. Zhou, C. Gao, L. Shi, and B. Chen, "Leapfrog formulation of the 3-D LOD-FDTD algorithm," IEEE Microwave and Wireless Components Letters, vol. 24, no. 3, pp. 137-139, 2014.

[14] J. A. Roden and S. D. Gedney, "Convolution PML (CPML): an efficient FDTD implementation of the CFS-PML for arbitrary media," Microwave and Optical Technology Letters, vol. 27, no. 5, pp. 334-339, 2000.

[15] E. L. Tan, "Fundamental schemes for efficient unconditionally stable implicit finite-difference time-domain methods," IEEE Transactions on Antennas and Propagation, vol. 56, no. 1, pp. 170-177, 2008.

[16] J. Shibayama, T. Oikawa, J. Yamauchi, and H. N. Life, "Efficient LOD-BOR-FDTD implementation based on a fundamental scheme," IEEE Photonics Technology Letters, vol. 24, no. 11, pp. 957-959, 2012.

[17] W. C. Tay and E. L. Tan, "Implementations of PMC and PEC boundary conditions for efficient fundamental ADI- and LODFDTD," Journal of Electromagnetic Waves and Applications, vol. 24, no. 4, pp. 565-573, 2010.

[18] J. Liu, G. Wan, J. Zhang, and X. Xi, "An effective CFS-PML implementation for cylindrical coordinate FDTD method," IEEE Microwave and Wireless Components Letters, vol. 22, no. 6, pp. 300-302, 2012. 


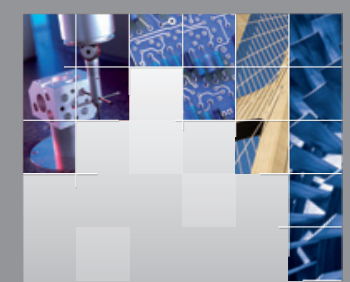

\section{Enfincering}
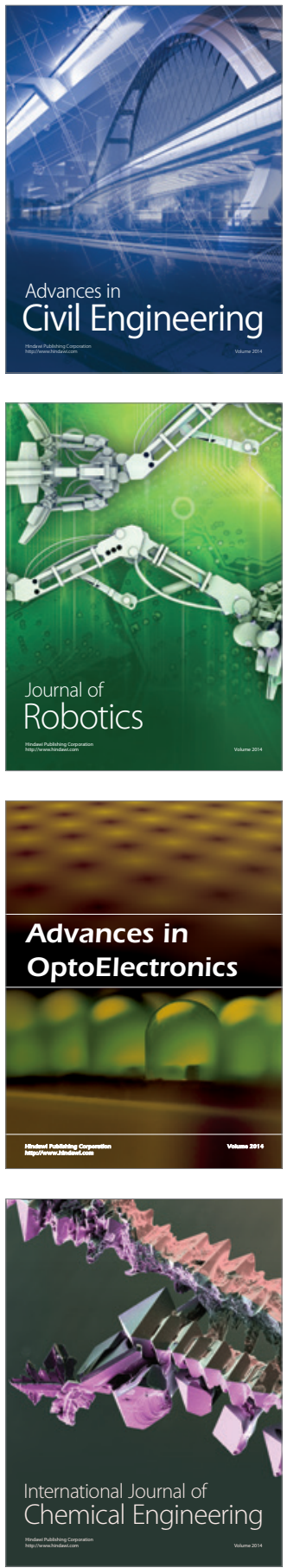

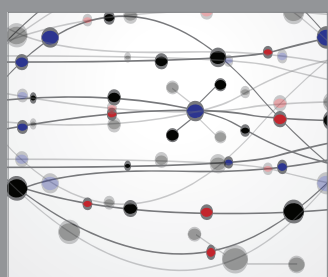

The Scientific World Journal

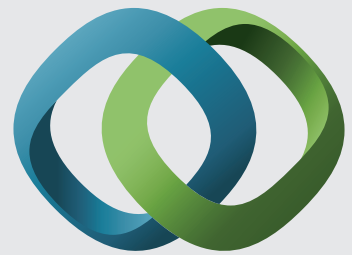

\section{Hindawi}

Submit your manuscripts at

http://www.hindawi.com
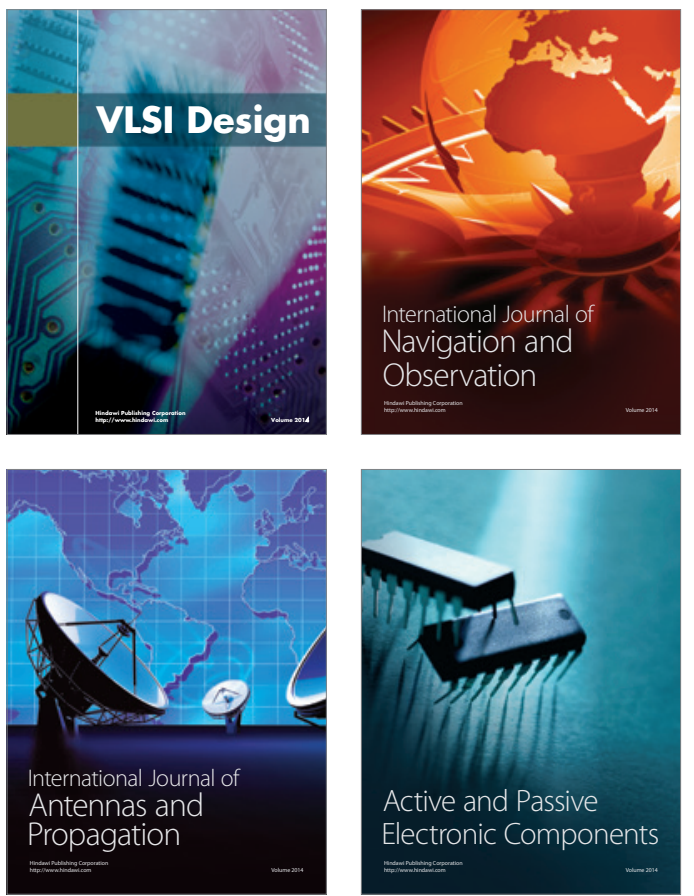
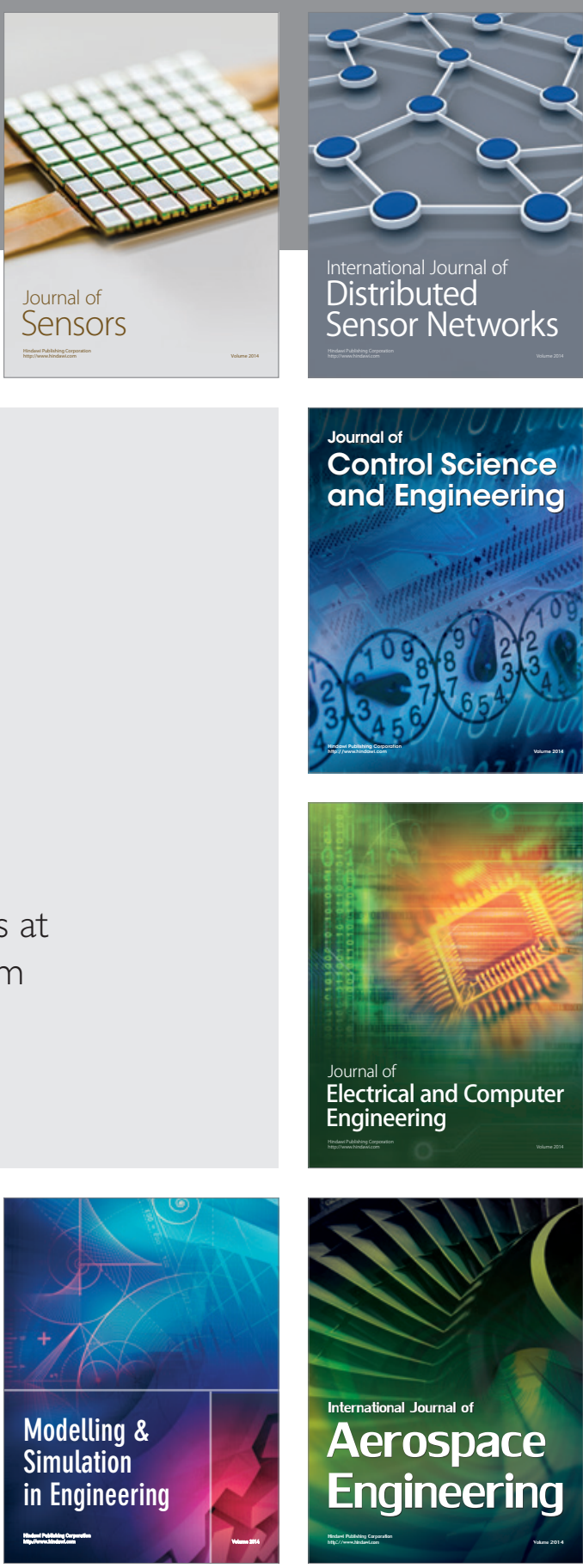

International Journal of

Distributed

Sensor Networks

Journal of

Control Science

and Engineering
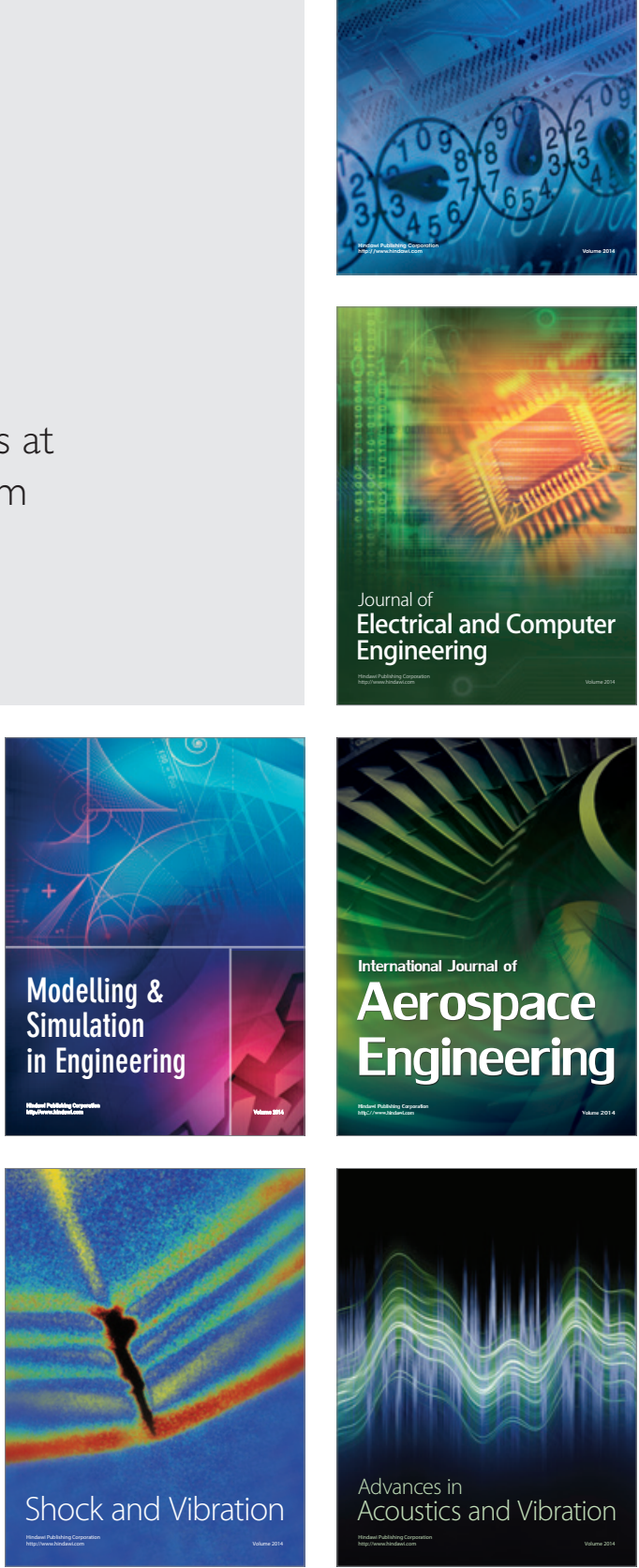